\title{
EUS fine-needle pancreatic core biopsy can determine eligibility for tumor-agnostic immunotherapy
}

\section{(ㄷ)(1) $\odot$}

Authors

Ferga C. Gleeson ${ }^{1}$, Michael J. Levy ${ }^{1}$, Anja C. Roden², Lisa A. Boardman ${ }^{1}$, Frank A. Sinicrope ${ }^{1,3}$, Robert R. McWilliams ${ }^{3}$, Lizhi Zhang ${ }^{2}$

Institutions

1 Division of Gastroenterology \& Hepatology, Mayo Clinic, Rochester, Minnesota, United States

2 Department of Laboratory Medicine and Pathology, Mayo Clinic, Rochester, Minnesota, United States

3 Division of Medical Oncology, Mayo Clinic, Rochester, Minnesota, United States

submitted 9.4.2018

accepted after revision 14.5.2018

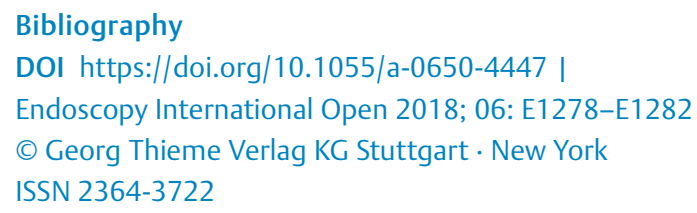

Corresponding author

Ferga C. Gleeson, MD, Mayo Clinic, Division of

Gastroenterology \& Hepatology, 200 1st, St SW, Rochester

MN 55905, USA

Fax: +1-507-266-3931

gleeson.ferga@mayo.edu

\section{ABSTRACT}

Background and study aims The US FDA recently approved a cancer treatment with pembrolizumab based upon the tumor biomarker status of deficient mismatch repair (dMMR) rather than a specific disease-based approach. We sought to determine if endoscopic ultrasound-guided fine-needle biopsy (EUS-FNB) could determine dMMR and quantification of PD-L1 expression to potentially guide the delivery of tumor agnostic immunotherapy.

Patients and methods Immunohistochemistry was performed on archived pancreas core biopsy specimens. Tumors with absent nuclear staining of DNA mismatch repair proteins represented dMMR. Tumors were considered to have any or high PD-L1 expression, if expressed in $\geq 1 \%$ or $\geq 50 \%$ of tumor cells.

Results Histologic specimen adequacy for MMR status assessment was satisfactory in $97.2 \%$ of tumors. dMMR and high PD-L1 expression was identified in $3 \%$ and $8.1 \%$ of the cohort.

Conclusion In the setting of tumor type agnostic immunotherapy, it is projected that at least $3 \%$ of malignant pancreas lesions will be sensitive to pembrolizumab and up to $8 \%$ sensitive to the family of immune checkpoint inhibitors. This highlights the expanding role of EUS-FNB in the field of precision immuno-oncology.

\section{Introduction}

Deficient DNA mismatch repair (MMR) results in a hypermutated phenotype characterized by microsatellite instability (MSI) and a higher burden of mutation-associated neoantigens that are targeted by the immune system. Deficient MMR and high frequency MSI (MSI-H) have been shown to be predictive biomarkers for immune checkpoint inhibitor drugs which block the programmed death protein-1/programmed death ligand-1 (PD-1/PD-L1) interaction between tumor cells and activated T cells. These agents include antibodies against PD-1 and PD-L1 that have significantly altered the treatment algorithm for several solid tumors. Presence and level of PD-L1 tumor expression are associated with responsiveness to these antibodies in certain malignancies that include advanced melanoma, non-small cell lung cancer, renal cell carcinoma, urothelial cancer and Hodgkin's lymphoma.

In 2017, the US Food and Drug Administration (FDA) granted accelerated approval to pembrolizumab (KEYTRUDA) for patients with unresectable or metastatic d-MMR solid tumors based upon an assessment of 15 cancer types, among which, $149 \mathrm{MSI}-\mathrm{H}$ patients were enrolled across 5 clinical trials [1]. A complete or partial response was experienced by $39.6 \%$ of patients, with responses lasting $\geq 6$ months in $78 \%$. Based upon these data, the FDA approved a cancer treatment for the first time based upon tumor biomarker status, rather than a specific disease-based approach.

Vanderwalde and colleagues recently published their experience evaluating a broad spectrum of 11,348 solid tumors noting the frequency of dMMR based on having MSI-H: PD-L1 ex- 
pression in endometrial cancer (17\%: 16.2\%), gastric adenocarcinoma (8.7\%: $18.5 \%)$, colorectal adenocarcinoma $(5.7 \%$ : $7.2 \%$ ), cholangiocarcinoma (2.3\%: $18.6 \%$ ), pancreatic ductal adenocarcinoma (PDAC) (1.2\%: $21.6 \%)$, renal cell carcinoma (0.6\%: $29.7 \%$ ) and melanoma (0\%: 42.3\%) [2]. The fact that $\mathrm{dMMR}$ is rarely present among PDAC patients was further demonstrated in a study of 833 surgically resected PDAC tumors revealing a frequency of $0.8 \%$, all of whom were patients found to have Lynch syndrome [3].

In an era of evolving tumor agnostic immunotherapy, we questioned if pancreatic endoscopic ultrasound-guided fineneedle biopsy (EUS FNB) provides sufficient material for dual immunohistochemistry (IHC) for dMMR and PD-L1 status evaluations. We sought to determine the prevalence of MSI-H status and quantification of PD-L1 expression to determine their utility in guiding disease-agnostic immunotherapy based upon biomarker status.

\section{Patients and methods}

IHC was performed on consecutive archived treatment-naïve formalin-fixed paraffin-embedded EUS pancreas core biopsy specimens (2001- 2017 IRB \# 17-006528). Briefly, 4- $\mu$ m-thick tissue sections were stained using the Ventana BenchMark XT automated slide-staining system using the following antibodies: Anti-PD-L1 (clone SP263, VENTANA, Tucson, AZ), MLH1 (clone G168-728, Cell Marque, Rocklin, California, United States), MSH2 (clone FE11, Biocare Medical, Concord, Massachusetts, United States), MSH6 (clone BC/44, Biocare Medical, Concord, Massachusetts, United States), and PMS2 (clone A164, Biocare Medical, Concord, M Massachusetts, United States). Antigen-antibody reactions were visualized using UltraView detection with diaminobenzidine as the chromogen.

A minimum of 100 viable tumor cells were required on one stained slide for the specimen to be considered adequate for evaluation. Positive PD-L1 expression was defined as membranous staining. The approximate percentage of positive tumor cells versus all tumor cells provided the Tumor Proportion Score (TPS). The specimen was considered to have PD-L1 expression if PD-L1 was expressed in $\geq 1 \%$ of tumor cells and a high level of expression if $\geq 50 \%$. Tumors were classified as dMMR if they exhibited absent nuclear staining of DNA mismatch repair proteins (MLH1, MSH2, MSH6, or PMS2).

\section{Results}

\section{Clinical demographics}

Thirty-nine treatment-naïve patients with either a primary or secondary pancreas malignancy who underwent EUS with FNB were assessed for histologic specimen adequacy for MMR and PD-L1 expression status. Age of the overall cohort was 71.2 \pm 10.2 years, $61.5 \%$ were male, and the overall mortality rate was $25.6 \%$ at 7 months (4.9-15.7) following EUS diagnosis. The study cohort was composed of 21 patients with PDAC who had previously reported PD-L1 expression analysis and with patient details as follows: $72.9 \pm 8.9$ years; $57 \%$ male; CA19-9 level $=143 \mathrm{U} / \mathrm{mL}(18-998) ; 57 \% 8^{\text {th }}$ AJCC Stage $\geq$ III $)$ [4]. The study also included 18 patients ( $69.3 \pm 11.6$ years; $66 \%$ male) with either a renal cell cancer (RCC) $(n=16)$ or melanoma that had metastasized to the pancreas $(n=2)$. Using either a 19G Trucut biopsy needle ( $\mathrm{n}=9$ patients) (Quick-Core Biopsy Needle, Cook Medical, Winston-Salem, North Carolina, United States) or a $22 \mathrm{G}$ Sharkcore FNB needle ( $\mathrm{n}=30$ patients) (SharkCore FNB needle, Medtronic, Dublin, Ireland), tumor tissue was sampled with an overall median tissue aggregate that was $19 \mathrm{~mm}$ (12$20)$ in length following $2(3-5)$ EUS-FNB passes.

\section{DNA mismatch repair status}

Among the 39 patients, 36 had specimen blocks available for retrieval from the tissue archive and 35 (97.2\%) patients (FNB passes 2 [3-5]) had histologic specimen adequacy to assess dMMR by IHC. One patient (2.9\%) displayed loss of MLH1PMS2 ( Fig. 1). The patient was a 64-year-old male with decompensated cirrhosis secondary to nonalcoholic steatohepatitis, a splenic artery pseudoaneurysm resulting in hemosuccus pancreaticus and previously resected colon cancer. He presented with cholestatic jaundice and an elevated CA19-9 (1,184 U/ $\mathrm{mL}$ ) as a consequence of a 4-cm EUS T4NOMO PDAC head mass. The additional immunostains performed on the tumor showed that the tumor cells were positive for CK7 and focally positive for CDX2 and CK20. This immunophenotype supports the diagnosis of a primary PDAC. He was started on FOLFIRINOX. He subsequently underwent germline testing which detected Lynch syndrome and received pembrolizumab. He had stable disease on therapy, but succumbed to vascular complications, presumably from his cirrhosis, 7.7 months following EUS.

\section{MMR status and PD-L1 expression}

Thirty-seven patients (94.9\%) had specimens that were satisfactory for PD-L1 expression assessment ( $>$ Fig. 2). Expression thresholds of $\geq 1 \%, \geq 5 \%, \geq 10 \%, \geq 25 \%$ and $\geq 50 \%$ expression in tumor cells were identified in 16 (43.2\%), 13 (35.1\%), 7 (18.9\%), 5 (13.5\%) and $3(8.1 \%)$ patients, respectively ( $\triangleright$ Fig.3). Tumor tissue from 33 patients underwent dual MSI and PD-L1 immunostaining ( $\triangleright$ Fig. 4). PD-L1 expression (> 1\%) with proficient $(p)$ MMR or dMMR status was identified in 13 (39.4\%) and 1 (3\%) patient, respectively. The tumor from the patient with Lynch syndrome had PD-L1 expression in $10 \%$ of tumor cells.

\section{Biomarker-based immunotherapy eligibility}

Based upon the prevalence of dMMR status, $3 \%$ of our patients would be eligible to receive pembrolizumab in the setting of a FDA-approved disease-agnostic immunotherapy approach. PDL1 expression was high (TPS $>50 \%$ ) in $8.1 \%$ of patients, suggesting that expression of this biomarker by their tumors may indicate potential sensitivity to immune checkpoint inhibitor therapy.

\section{Discussion}

Among a unique patient cohort, we determined that a paired evaluation of MMR status and PD-L1 expression by IHC is possible using specimens obtained with EUS-FNB. Our findings de- 


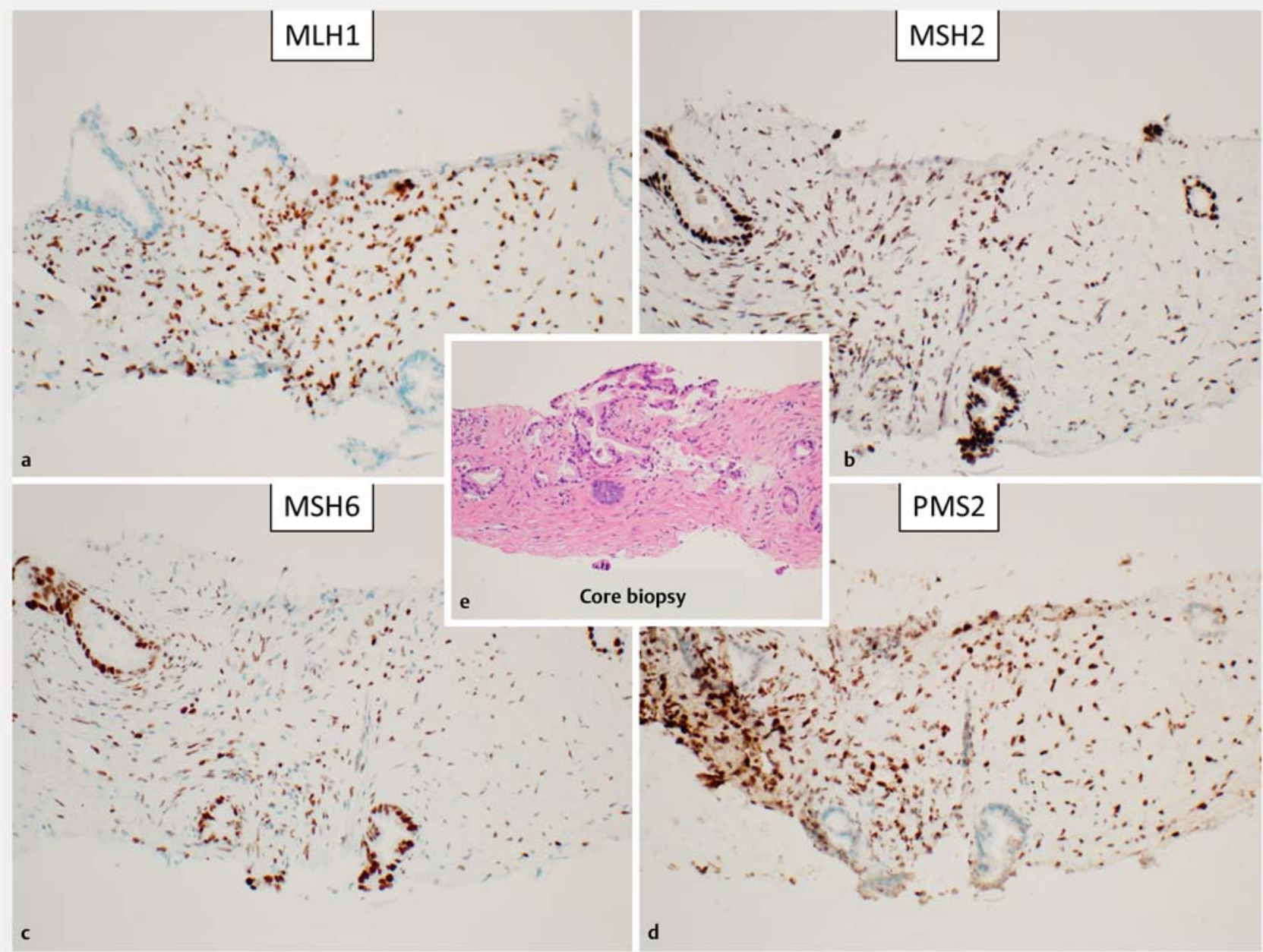

Fig. 1a-e Loss of MLH1-PMS2 protein expression in a PDAC EUS-FNB specimen.

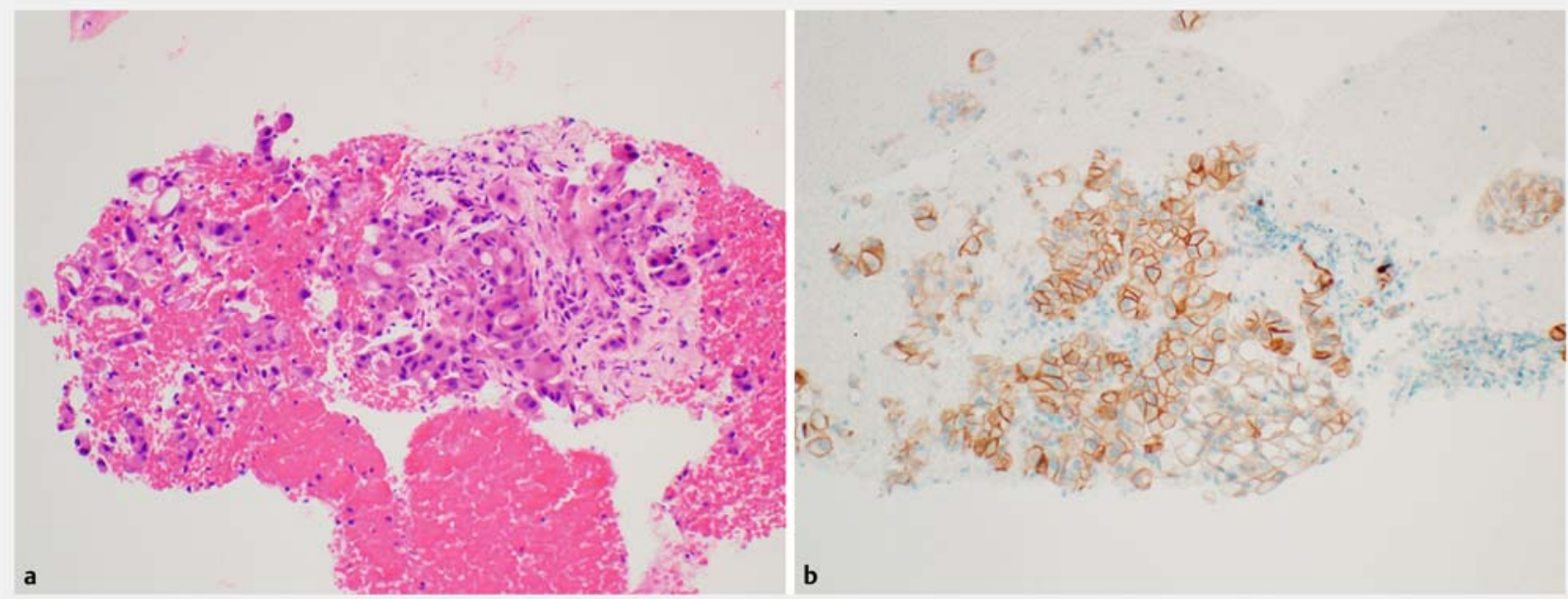

Fig. 2 a EUS-FNB malignant melanoma metastasis to the pancreas with b PD-L1 expression >90\%. 


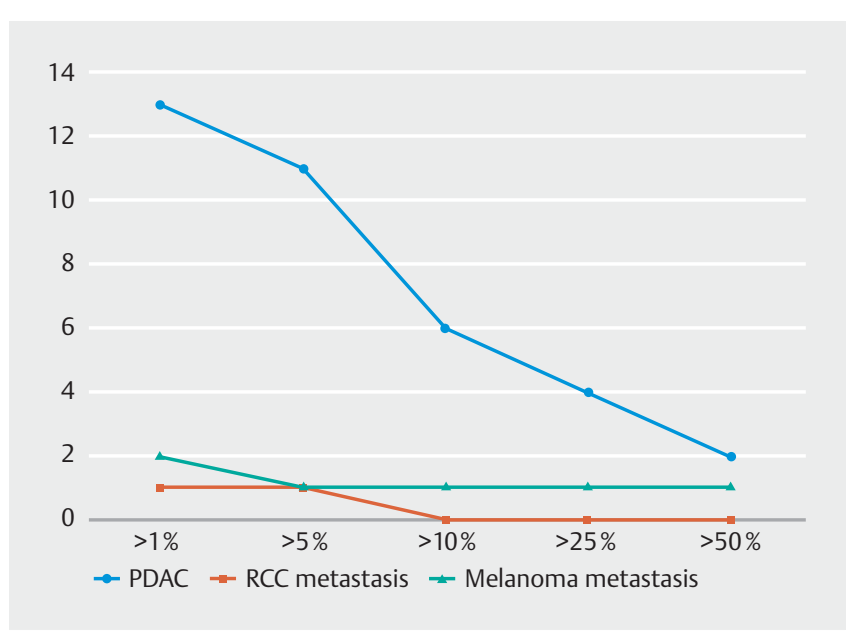

- Fig. 3 Patient prevalence and quantification of PD-L1 expression positivity for EUS-FNB specimens of treatment-naïve PDAC, clear cell renal cell carcinoma and malignant melanoma.

monstrate the capability of using tissues obtained with EUSFNB for molecular classification of treatment-naïve solid pancreas masses by identifying biomarkers that may guide use of checkpoint inhibitor therapy. The $3 \%$ prevalence of dMMR in our cohort indicates that patients with these tumors are candidates for treatment with pembrolizumab. Furthermore, $8.1 \%$ of evaluated tumors had a high level of expression of PD-L1 (TPS $>50 \%$ ), which in certain tumor types is associated with responsiveness to checkpoint inhibitor therapy.

Following much debate, it has been established that endoscopic ultrasound-guided fine-need aspiration and EUS-FNB needles yield a similar satisfactory specimen for diagnostic purposes, with the caveat that fewer FNB passes are required to achieve such a yield [5-10]. However, when tissue for companion diagnostic or prognostic tumor information is required for integration of molecular analyses into clinical decision-making, the optimal needle landscape changes depending upon the specific objective. Options for use of tissue specimens include IHC, genotyping using next-generation sequencing (NGS), or establishing tumor organoids in a research setting. PDACs demonstrate only $5 \%$ to $20 \%$ neoplastic cellularity and are characterized by a prominent desmoplastic reaction considered to be a hostile tumor microenvironment (TME) for subsequent therapy $[11,12]$. Cytology smears are thought to be superior for multiplex gene panel NGS evaluations, as their overall cellularity, tumor fraction, and sequencing metrics are considered to be superior to that of FNB, yet, successful preclinical disease models to include organoid development require that EUS-FNB deliver tumor cells and the corresponding TME [13-15].

As endosonographers, while we try to navigate the provision of satisfactory material for ancillary molecular analysis to improve cytologic adequacy and identify predictors of prognosis and chemosensitivity, we are now poised to assist in patient selection for precision immuno-oncology. There are a number of barriers to the utility of immunotherapy in PDAC as the mutational burden is low; the TME is characterized by dense stroma with very few $\mathrm{T}$ cells, collectively creating a "cold" or non-im-

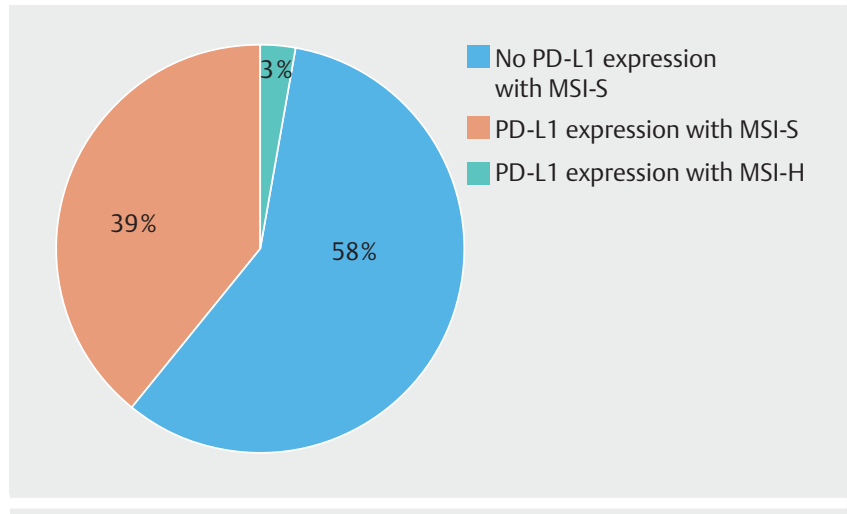

- Fig. 4 Dual MSI and PD-L1 IHC assessment yield.

munogenic environment and potentially making such tumors less responsive to checkpoint inhibitors. Yet, there is much need for enhanced tissue access and sampling for analysis to utilize biomarkers in patients with solid tumors, including PDAC, which can determine eligibility for checkpoint inhibitor therapy.

To date, the MMR/MSI status of pancreatic lesions has only been evaluated in surgically resected specimens, which represent a small proportion of patients that have earlier, less advanced disease, as patients with more advanced disease are no longer surgical candidates. MMR and PD-L1 status may be influenced by prior neoadjuvant or adjuvant therapy as has been observed in both breast and colon cancer analyses [16,17]. Therefore, for primary pancreatic masses to include PDAC, it may be more desirable to perform such analysis on treatment-naïve specimens for which EUS-FNB is ideally suited to establish the molecular signature. For secondary pancreatic metastasis, we evaluated RCC and melanoma treatment-naïve lesions, which is the ideal scenario. However, from a practical perspective, patients with these lesions have usually undergone prior treatment that requires careful consideration of the molecular testing.

\section{Conclusion}

In conclusion, we highlight that our early experience with dual MMR IHC and PD-L1 expression analysis shows that it is technically feasible to assess biomarker-based immunotherapy eligibility with EUS-FNB specimens from solid primary and secondary pancreatic masses. Based upon the prevalence of $d M M R$ and high PD-L1 expression, $3 \%$ of the treatment-naïve cohort is a candidate for checkpoint inhibitor therapy and high PD-L1 expression may provide additional information regarding sensitivity to immunotherapy depending upon tumor type.

\section{Competing interests}

None 


\section{References}

[1] US Food and Drug Administration. FDA approves first cancer treatment for any solid tumor with a specific genetic feature. https:// www.fda.gov/newsevents/newsroom/pressannouncements/ ucm560167.htm

[2] Vanderwalde A, Spetzler D, Xiao $\mathrm{N}$ et al. Microsatellite instability status determined by next-generation sequencing and compared with PD-L1 and tumor mutational burden in 11,348 patients. Cancer Med 2018; 7: $746-756$

[3] Hu ZI, Shia J, Stadler ZK et al. Evaluating mismatch repair deficiency in pancreatic adenocarcinoma: challenges and recommendations. Clin Cancer Res 2018; 24: 1326-1336

[4] Gleeson FC, Zhang L, Roden AC et al. EUS fine-needle biopsy can determine PD-L1 immune biomarker status in treatment naïve pancreatic ductal adenocarcinoma. Clin Gastroenterol Hepatol 2018; 3 : doi:10.1016/j.cgh.2018.01.001

[5] Lee BS, Cho CM, Jung MK et al. comparison of histologic core portions acquired from a core biopsy needle and a conventional needle in solid mass lesions: a prospective randomized trial. Gut Liver 2017; 15: $559-566$

[6] Lee YN, Moon JH, Kim HK et al. Core biopsy needle versus standard aspiration needle for endoscopic ultrasound-guided sampling of solid pancreatic masses: a randomized parallel-group study. Endoscopy 2014; 46: 1056 - 1062

[7] Tian L, Tang AL, Zhang L et al. Evaluation of 22G fine-needle aspiration (FNA) versus fine-needle biopsy (FNB) for endoscopic ultrasoundguided sampling of pancreatic lesions: a prospective comparison study. Surg Endosc 2018; 32: 3533-3539

[8] Hedenström P, Demir A, Khodakaram K et al. EUS-guided reverse bevel fine-needle biopsy sampling and open tip fine-needle aspiration in solid pancreatic lesions - a prospective, comparative study. Scand J Gastroenterol 2018; 53: 231-237
[9] Strand DS, Jeffus SK, Sauer BG et al. EUS-guided 22-gauge fine-needle aspiration versus core biopsy needle in the evaluation of solid pancreatic neoplasms. Diagn Cytopathol 2014; 42: 751 - 758

[10] Bang JY, Hebert-Magee S, Trevino J et al. Randomized trial comparing the 22-gauge aspiration and 22-gauge biopsy needles for EUS-guided sampling of solid pancreatic mass lesions. Gastrointest Endosc 2012; 76: $321-327$

[11] lacobuzio-Donahue CA, Ryu B, Hruban RH et al. Exploring the host desmoplastic response to pancreatic carcinoma: gene expression of stromal and neoplastic cells at the site of primary invasion. Am J Pathol 2002; 160: 91 - 99

[12] Wood LD, Hruban RH. Pathology and molecular genetics of pancreatic neoplasms. Cancer J 2012; 18: 492 - 501

[13] Roy-Chowdhuri S, Chen H, Singh RR et al. Concurrent fine needle aspirations and core needle biopsies: a comparative study of substrates for next-generation sequencing in solid organ malignancies. Mod Pathol 2017; 30: 499-508

[14] Wani S, Muthusamy VR, McGrath CM et al. AGA White Paper: optimizing endoscopic ultrasound-guided tissue acquisition and future directions. Clin Gastroenterol Hepatol 2018; 16: 318-327

[15] Tiriac H, Bucobo JC, Tzimas D et al. Successful creation of pancreatic cancer organoids by means of EUS-guided fine-needle biopsy sampling for personalized cancer treatment. Gastrointest Endosc 2018; 87: $1474-1480$

[16] Goldstein JB, Wu W, Borras E et al. Can microsatellite status of colorectal cancer be reliably assessed after neoadjuvant therapy? Clin Cancer Res 2017; 1: 5246- 5254

[17] Pelekanou V, Carvajal-Hausdorf DE, Altan M et al. Effect of neoadjuvant chemotherapy on tumor-infiltrating lymphocytes and PD-L1 expression in breast cancer and its clinical significance. Breast Cancer Res 2017; 7: 91 